\title{
Historical Data Analysis
}

National Cancer Institute

\section{Source}

National Cancer Institute. Historical Data Analysis. NCI Thesaurus. Code C139455.

The investigation involved the analysis of historical adverse events data of the actual device involved in the adverse event and/or of products from the same and/or different batches/lots. 